DESY 05-089

\title{
The Gravitino in Gaugino Mediation
}

\author{
Wilfried Buchmüller ${ }^{\mathrm{a}}$, Koichi Hamaguchi ${ }^{\mathrm{b}}$ and Jörn Kersten ${ }^{\mathrm{c}}$ \\ Deutsches Elektronen-Synchrotron DESY, 22603 Hamburg, Germany
}

\begin{abstract}
We show that for gaugino mediated supersymmetry breaking the gravitino mass is bounded from below. For a size of the compact dimensions of order the unification scale and a cutoff given by the higher-dimensional Planck mass, we find $m_{3 / 2} \gtrsim 10 \mathrm{GeV}$. In a large domain of parameter space, the gravitino is the lightest superparticle with a scalar $\tilde{\tau}$-lepton as the next-to-lightest superparticle.
\end{abstract}

\footnotetext{
${ }^{a}$ Email: wilfried.buchmueller@desy.de

${ }^{\mathrm{b}}$ Email: koichi.hamaguchi@desy.de

${ }^{\mathrm{c}}$ Email: joern.kersten@desy.de
} 


\section{Introduction}

In supersymmetric (SUSY) models with R-parity conserved, the lightest superparticle (LSP) is stable and plays a key role in cosmology as well as in collider physics. The most widely studied LSP candidate is the neutralino, which is a linear combination of the gauginos and higgsinos. There is however another candidate, the gravitino, which is always present once SUSY is extended to a local symmetry leading to supergravity.

As a neutral and stable particle, the gravitino LSP is a natural cold dark matter candidate [1]. ${ }^{1}$ In the early universe gravitinos are produced by thermal scatterings after inflation $[3,4,5]$. Generically, their relic abundance exceeds the observed cold dark matter density unless the reheating temperature $T_{R}$ is sufficiently small. However, several mechanisms have been proposed which avoid this constraint and yield the correct dark matter density even for large $T_{R}[6,7]$. This also removes an obstacle for thermal leptogenesis [8], which requires $T_{R} \gtrsim 2 \cdot 10^{9} \mathrm{GeV}$ [9]. Such a high reheating temperature is disfavored for a non-LSP gravitino, since its decays would alter the light element abundances produced by big-bang nucleosynthesis $[10,4,11]$. Even for a gravitino LSP, the late time decay of the next-to-lightest superparticle (NLSP) may cause cosmological problems. Recent analyses show that a stau NLSP is allowed for a gravitino mass $\lesssim 10-100 \mathrm{GeV}$, while for a neutralino NLSP the bound is severer [12]. Gravitino dark matter can also be realized via non-thermal production from decays of the NLSP [13].

If the long-lived NLSP is a charged particle, such as the stau, it can be collected and studied in detail at the LHC and the ILC, which allows to explore various aspects of new physics $[14,15]$. Particularly interesting are gravitino masses in the range $10-100 \mathrm{GeV}$, where one may be able to measure the Planck scale as well as the gravitino spin [14].

The theoretical predictions for the gravitino mass and the nature of the LSP depend on the mechanism of SUSY breaking. In models with gauge mediation, the gravitino is usually much lighter than $1 \mathrm{GeV}$ [16], so that it clearly becomes the LSP. For gravity mediation, its mass is of the same order as the masses of scalar quarks and leptons, i.e. $100 \mathrm{GeV}-1 \mathrm{TeV}$ [17]. Whether it is the LSP or not depends on the details of the model. On the other hand, anomaly mediation predicts a very heavy gravitino [18], which cannot be the LSP.

In this Letter we discuss the gravitino mass for gaugino mediated SUSY breaking [19], which is one of the simplest mechanisms solving the SUSY flavor problem (cf. also [20]). The role of the gravitino in this context has not been studied in detail in the literature. We use naive dimensional analysis (NDA) to derive a lower bound on the gravitino to gaugino mass ratio. From this we conclude that the gravitino mass is typically larger than about $10 \mathrm{GeV}$. Therefore, it can be the LSP. In this case the NLSP is naturally the stau. Together with the relatively large gravitino mass, this has exciting consequences for cosmology and collider physics.

\footnotetext{
${ }^{1} \mathrm{~A} \mathrm{keV} \mathrm{gravitino} \mathrm{as} \mathrm{dominant} \mathrm{component} \mathrm{of} \mathrm{dark} \mathrm{matter} \mathrm{as} \mathrm{discussed} \mathrm{in} \mathrm{[1]} \mathrm{is} \mathrm{now} \mathrm{disfavored} \mathrm{by} \mathrm{the}$ matter power spectrum (cf. [2]).
} 


\section{Gaugino Mediation}

We consider a theory with $D$ dimensions and 4-dimensional branes located at positions $y_{i}$ in the compact dimensions. Coordinates $x$ denote the usual 4 dimensions, while $y$ refer to the compact dimensions. In models with gaugino mediated SUSY breaking [19], the gauge superfields live in the bulk, while the scalar responsible for SUSY breaking (contained in the chiral superfield $S$ ) lives on the 4-dimensional brane $i=1$. The part of the Lagrangian relevant for gaugino masses is

$$
\begin{aligned}
\mathscr{L}_{D}= & \frac{1}{4 g_{D}^{2}} \int \mathrm{d}^{2} \theta W^{a} W^{a}+\text { h.c. }+ \\
& +\delta^{(D-4)}\left(y-y_{1}\right) \int \mathrm{d}^{4} \theta S^{\dagger} S+ \\
& +\delta^{(D-4)}\left(y-y_{1}\right) \frac{h}{4 \Lambda} \int \mathrm{d}^{2} \theta S W^{a} W^{a}+\text { h.c. }
\end{aligned}
$$

where $W^{a}$ is the field strength superfield, $h$ is a dimensionless coupling and $\Lambda$ is the cutoff of the theory. All fields are 4D $N=1$ superfields. Bulk fields depend on the coordinates $y$. For details of the formalism, see [21]. Additional fields required by the higher-dimensional SUSY are present but not explicitly included in the Lagrangian, since they are not relevant for our discussion.

A vacuum expectation value (vev) $F_{S}$ for the $F$-term of $S$ breaks SUSY and leads to the gaugino mass

$$
m_{1 / 2}=\frac{g_{4}^{2} h F_{S}}{2 \Lambda}
$$

at the compactification scale. The gravitino mass is given by [17]

$$
m_{3 / 2}=\frac{1}{\sqrt{3}} \frac{F_{S}}{M_{4}}
$$

where $M_{4} \simeq 2.4 \cdot 10^{18} \mathrm{GeV}$ is the 4-dimensional (reduced) Planck mass. This relation is valid if the vev of $F_{S}$ is the only source of SUSY breaking. If there are further sources, the gravitino becomes heavier.

\section{Constraints from Naive Dimensional Analysis}

We want the effective Lagrangian (1) to be valid up to a cutoff scale $\Lambda$. This requires that the couplings at the compactification scale do not exceed upper bounds which can be estimated by means of 'naive dimensional analysis' [22]. In general, one rewrites the $D$-dimensional Lagrangian with bulk fields $\Phi(x, y)$ and brane fields $\phi_{i}(x)$ on the $i$ th brane,

$$
\mathscr{L}_{D}=\mathscr{L}_{\text {bulk }}(\Phi(x, y))+\sum_{i} \delta^{D-4}\left(y-y_{i}\right) \mathscr{L}_{i}\left(\Phi(x, y), \phi_{i}(x)\right)
$$


in terms of dimensionless fields $\hat{\Phi}(x, y)$ and $\hat{\phi}_{i}(x)$, and the cutoff $\Lambda$, so that

$$
\mathscr{L}_{D}=\frac{\Lambda^{D}}{\ell_{D} / C} \hat{\mathscr{L}}_{\text {bulk }}(\hat{\Phi}(x, y))+\sum_{i} \delta^{D-4}\left(y-y_{i}\right) \frac{\Lambda^{4}}{\ell_{4} / C} \hat{\mathscr{L}}_{i}\left(\hat{\Phi}(x, y), \hat{\phi}_{i}(x)\right) .
$$

Here the Lagrangians $\hat{\mathscr{L}}$ have kinetic terms of the form $\hat{\mathscr{L}}=\left(\frac{\partial}{\Lambda} \hat{\Phi}\right)^{2}+\ldots$ for scalars, and analogously for other fields. If the kinetic terms of the original Lagrangian (4) are canonical with respect to $\Phi$ and $\phi_{i}$, the rescaling of bosonic bulk and brane fields reads

$$
\Phi(x, y)=\left(\frac{\Lambda^{D-2}}{\ell_{D} / C}\right)^{1 / 2} \hat{\Phi}(x, y) \quad, \quad \phi_{i}(x)=\left(\frac{\Lambda^{2}}{\ell_{4} / C}\right)^{1 / 2} \hat{\phi}_{i}(x) .
$$

For non-canonical kinetic terms in Eq. (4), the field rescaling has to be adjusted so that Eq. (5) is obtained. The geometrical loop factor

$$
\ell_{D}=2^{D} \pi^{D / 2} \Gamma(D / 2)
$$

grows rapidly with the number of dimensions: $\ell_{4}=16 \pi^{2}, \ell_{5}=24 \pi^{3}, \ell_{6}=128 \pi^{3}$ etc. The factor $C$ accounts for the multiplicity of fields in loop diagrams for a non-Abelian gauge group $G$. We choose $C=C_{2}(G)$, i.e. $C=5$ for $\mathrm{SU}(5)$ and $C=8$ for $\mathrm{SO}(10)$.

The combination $C / \ell_{D}$ gives the typical geometrical suppression of loop diagrams. This suppression is canceled by the factors $\ell_{D} / C$ and $\ell_{4} / C$ in front of the Lagrangians $\hat{\mathscr{L}}$ in Eq. (5). Consequently, all loops will be of the same order of magnitude, provided that all couplings are $\mathscr{O}(1)$. Thus, according to the NDA recipe the effective $D$-dimensional theory remains weakly coupled up to the cutoff $\Lambda$, if the dimensionless couplings in Eq. (5) are smaller than one.

As an example, consider the $D$-dimensional gauge coupling

$$
\frac{V_{D-4}}{g_{D}^{2}}=\frac{1}{g_{4}^{2}},
$$

where $V_{D-4}$ is the volume of the compact dimensions. From the dimensionless covariant derivative,

$$
\hat{D}_{\mu}=\frac{\partial_{\mu}}{\Lambda}-\frac{i g_{D} A_{\mu}}{\Lambda}=\frac{\partial_{\mu}}{\Lambda}-i g_{D}\left(\frac{\Lambda^{D-4}}{\ell_{D} / C}\right)^{1 / 2} \hat{A}_{\mu}
$$

one reads off

$$
g_{D}\left(\frac{\Lambda^{D-4}}{\ell_{D} / C}\right)^{1 / 2}<1
$$

For a given cutoff this constrains the gauge coupling. Conversely, knowing the gauge coupling at the compactification scale, $g_{4}^{2}$, one obtains an upper bound on the cutoff,

$$
\Lambda<\Lambda_{\text {gauge }}=\left(\frac{\ell_{D} / C}{g_{4}^{2}}\right)^{\frac{1}{D-4}} M_{c}
$$




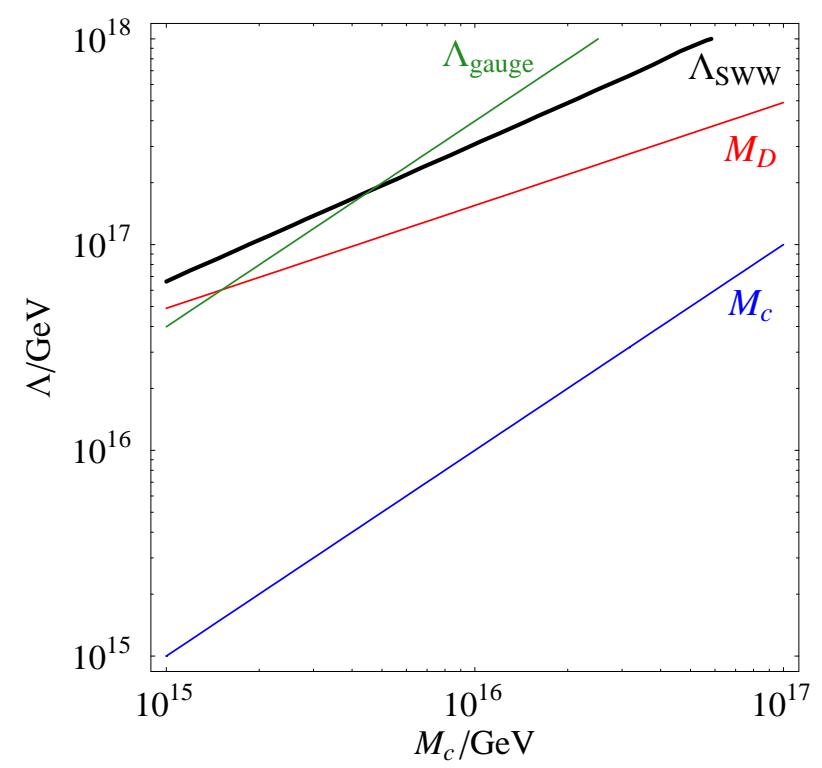

Figure 1: Relevant scales $(D=6): \Lambda_{S W W}$ for $m_{1 / 2}=m_{3 / 2}$ and $C=5$, ignoring the running of the gaugino masses, $\Lambda_{\text {gauge }}$ for $g_{4}^{2}=\frac{1}{2}, D$-dimensional Planck scale, and the lower limit $\Lambda>M_{c}$.

where we have defined

$$
M_{c}=\left(\frac{1}{V_{D-4}}\right)^{\frac{1}{D-4}} .
$$

For $M_{c}$ close to the unification scale, one has $g_{4}^{2} \simeq \frac{1}{2}$.

The cutoff $\Lambda_{\text {gauge }}$ can be compared with the $D$-dimensional Planck scale $M_{D}$ where quantum gravity effects are expected to become important,

$$
M_{D}=\left(M_{c}^{D-4} M_{4}^{2}\right)^{\frac{1}{D-2}},
$$

as shown in Fig. 1 for $D=6$. We require $\Lambda<M_{D}$, which turns out to be more restrictive than $\Lambda<\Lambda_{\text {gauge }}$, unless $M_{c}$ is very small.

\section{Application to Gaugino Mediation}

We can now apply the NDA prescription to gaugino mediation, which will lead to an upper bound on the gaugino masses. The field strength superfield has to be rescaled as (cf. Eq. (1) and (5))

$$
W^{a}=\left(\frac{\Lambda^{D-1}}{\ell_{D} / C}\right)^{1 / 2} g_{D} \hat{W}^{a} .
$$


Since $\mathrm{d} \theta$ has mass dimension $1 / 2$, it also has to be divided by the corresponding power of the cutoff to obtain a dimensionless expression. We then arrive at the Lagrangian

$$
\begin{aligned}
\mathscr{L}_{D}= & \frac{\Lambda^{D}}{\ell_{D} / C} \frac{1}{4} \int \frac{\mathrm{d}^{2} \theta}{\Lambda} \hat{W}^{a} \hat{W}^{a}+\text { h.c. }+ \\
& +\delta^{(D-4)}\left(y-y_{1}\right) \frac{\Lambda^{4}}{\ell_{4} / C} \int \frac{\mathrm{d}^{4} \theta}{\Lambda^{2}} \hat{S}^{\dagger} \hat{S}+ \\
& +\delta^{(D-4)}\left(y-y_{1}\right) \frac{\Lambda^{4}}{\ell_{4} / C} \frac{g_{D}^{2} h \sqrt{\ell_{4} C} \Lambda^{D-4}}{\ell_{D}} \frac{1}{4} \int \frac{\mathrm{d}^{2} \theta}{\Lambda} \hat{S} \hat{W}^{a} \hat{W}^{a}+\text { h.c. }
\end{aligned}
$$

The requirement that all couplings ${ }^{2}$ be smaller than one implies

$$
\frac{g_{D}^{2} h \sqrt{\ell_{4} C} \Lambda^{D-4}}{\ell_{D}}<1
$$

Using the relations (8) and (12), and $\ell_{4}=16 \pi^{2}$, one then obtains an upper bound on the coupling $h$,

$$
h<\frac{\ell_{D}}{4 \pi \sqrt{C} g_{4}^{2}}\left(\frac{M_{c}}{\Lambda}\right)^{D-4}
$$

which translates into an upper bound on the gaugino mass (cf. Eq. (2)):

$$
m_{1 / 2}<\frac{\ell_{D} F_{S}}{8 \pi \sqrt{C} \Lambda}\left(\frac{M_{c}}{\Lambda}\right)^{D-4} .
$$

Note that there is no lower bound on the gaugino mass. The upper bound becomes weaker if the cutoff $\Lambda$ is lowered. Together with Eq. (3), Eq. (18) yields a lower bound on the mass ratio

$$
\frac{m_{3 / 2}}{m_{1 / 2}}>\frac{8 \pi \sqrt{C}}{\sqrt{3} \ell_{D}}\left(\frac{\Lambda}{M_{c}}\right)^{D-4} \frac{\Lambda}{M_{4}} .
$$

For a fixed gravitino to gaugino mass ratio, Eq. (19) yields again an upper bound on the cutoff $\Lambda$,

$$
\Lambda<\Lambda_{S W W}=\left(\frac{\sqrt{3} \ell_{D}}{8 \pi \sqrt{C}} M_{4} M_{c}^{D-4} \frac{m_{3 / 2}}{m_{1 / 2}}\right)^{\frac{1}{D-3}}
$$

which is compared in Fig. 1 with $\Lambda_{\text {gauge }}$ and $M_{D}$ as a function of $M_{c}$.

Let us now discuss the lower bound (19) on the gravitino to gaugino mass ratio for a given cutoff $\Lambda$. For the minimal value $\Lambda=M_{c}$, one obtains the absolute lower bound $m_{3 / 2} / m_{1 / 2}>8 \pi \sqrt{C} /\left(\sqrt{3} \ell_{D}\right) M_{c} / M_{4}$. However, this corresponds to the extreme case

\footnotetext{
${ }^{2}$ Note that this applies to the couplings of canonically normalized fields. Hence, the factor $\frac{1}{4}$ in the last line of Eq. (15) is not part of the coupling.
} 


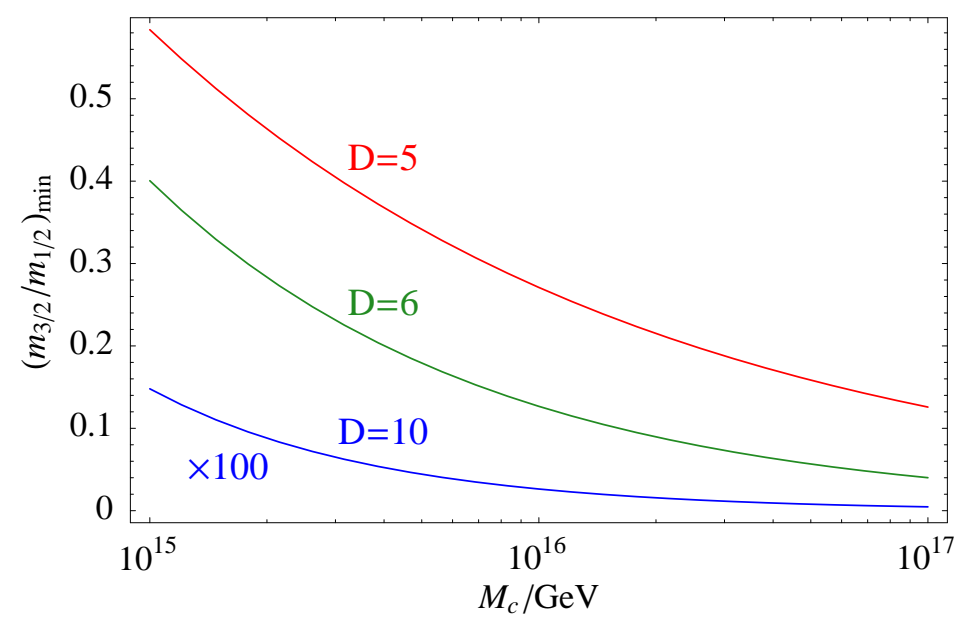

Figure 2: Lower bound on the ratio of the gravitino and the gaugino mass (valid at the compactification scale) for a cutoff $\Lambda=M_{D}, C=5$, and $D=5,6,10$. The $D=10$ result is multiplied by a factor of 100 .

where the effective theory described by the Lagrangian (1) becomes non-perturbative immediately above $M_{c}$. In the following, we choose the cutoff to equal the $D$-dimensional Planck scale for concreteness. The minimal mass ratio is then a function of the number of dimensions and the compactification scale,

$$
\left(\frac{m_{3 / 2}}{m_{1 / 2}}\right)_{\min }=\frac{8 \pi \sqrt{C}}{\sqrt{3} \ell_{D}}\left(\frac{M_{4}}{M_{c}}\right)^{\frac{D-4}{D-2}} .
$$

It is shown in Fig. 2 for $D=5,6,10$ and compactification scales between $10^{15} \mathrm{GeV}$ and $10^{17} \mathrm{GeV}$. As we are assuming compact dimensions of equal size, the $D=10$ example appears less favored [23] and should only be considered as a limiting case for illustration purposes. Note that here $m_{1 / 2}$ is the value of the gaugino mass at the compactification scale. The running to low energies typically decreases the mass of the lightest gaugino by a factor of about 0.4. We have not included this correction here, since it is modeldependent. From the figure we see that the gravitino can be the LSP, if the gaugino mass is sufficiently close to its upper bound from NDA. However, it cannot be much lighter than the neutralinos for $D=5$ and $D=6$. This also means that a gravitino LSP becomes unlikely if the theory is only weakly coupled at the cutoff.

Fixing $m_{1 / 2}$, we find a lower bound for $m_{3 / 2}$, which is shown in Fig. 3 for a gluino mass (at low energy) of $1 \mathrm{TeV}$. For $D=6$ and $M_{c}=10^{17} \mathrm{GeV}$, we obtain $m_{3 / 2}>$ $17 \mathrm{GeV}$. This can be considered a typical lower bound in gaugino mediation. Hence, experimental implications for a much lighter gravitino would disfavor this mechanism of SUSY breaking. To realize smaller gravitino masses, one can lower $m_{1 / 2}$ or the cutoff scale, or increase the number of extra dimensions. Further possibilities include the group theory factor $C$, which could be smaller if the GUT symmetry was broken on the brane where $S$ is located.

Another important aspect of the scenario is the mass of the lighter stau. In gaugino mediation, it is very small at the compactification scale, so that its dominant contribution 


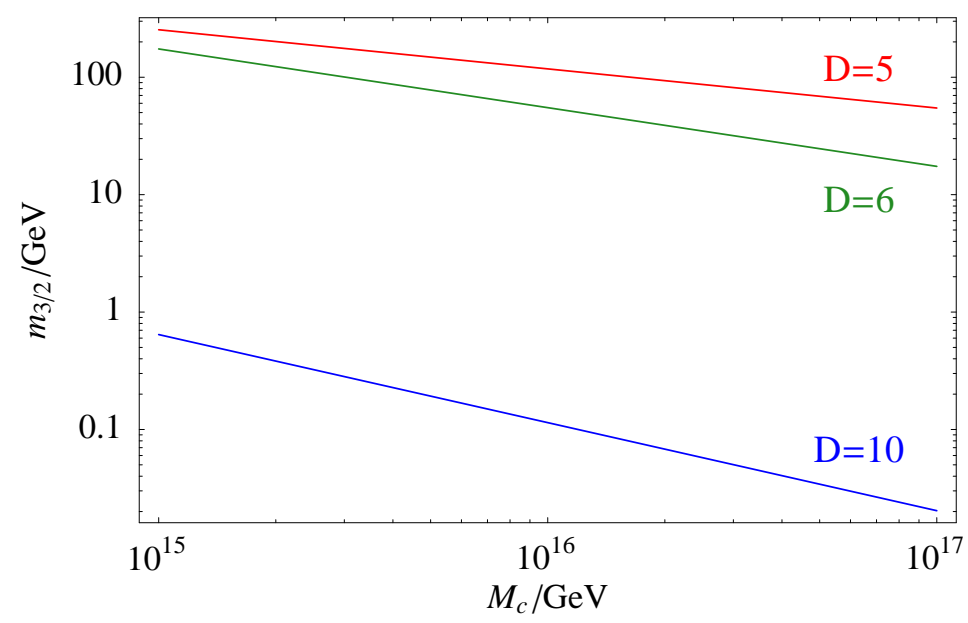

Figure 3: Lower bound on the gravitino mass for $M_{3}(1 \mathrm{TeV})=1 \mathrm{TeV}, \Lambda=M_{D}, C=5$, and $D=5,6,10$.

is due to the running to low energies. It is determined by the renormalization group equations of the right-handed stau mass squared [24],

$$
16 \pi^{2} \mu \frac{\mathrm{d}}{\mathrm{d} \mu} m_{\tilde{\tau}_{\mathrm{R}}}^{2}=4 y_{\tau}^{2}\left(m_{H_{d}}^{2}+m_{\tilde{\tau}_{\mathrm{L}}}^{2}+m_{\tilde{\tau}_{\mathrm{R}}}^{2}\right)+4 a_{\tau}^{2}-\frac{24}{5} g_{1}^{2} M_{1}^{2},
$$

and of the other parameters of the theory. In order to demonstrate the typical order of magnitude, Fig. 4 shows the ratio of the $\tilde{\tau}_{\mathrm{R}}$ and the bino mass at the electroweak scale calculated in a crude approximation: Mixing has been neglected, and only the terms proportional to $M_{1}^{2}$ and $m_{\tilde{\tau}_{\mathrm{R}}}^{2}$ in Eq. (22) have been taken into account. We have chosen $\tan \beta=10, m_{1 / 2}\left(M_{c}\right)=400 \mathrm{GeV}$ and $m_{H_{u}}^{2}\left(M_{c}\right)=m_{H_{d}}^{2}\left(M_{c}\right)=0$. From the figure we see that in this case the $\tilde{\tau}$ is typically lighter than the $\tilde{B}$, but not by a large factor, so that it can easily be heavier than the gravitino. A comparison with the full two-loop calculations [25] shows that the accuracy of the approximation is reasonable for small $\tan \beta$ (and $m_{H_{u}}^{2}=m_{H_{d}}^{2}=0$ at $M_{c}$ ) but quickly worsens for values larger than about 20 . Then, the neglected effects are important and the actual stau mass becomes significantly lighter than the estimate. We have also neglected the modifications to the running above the GUT scale for large $M_{c}$, which tend to make the stau heavier [26].

\section{Conclusions}

We have studied constraints on gaugino and gravitino masses in models with gaugino mediated SUSY breaking. Based on naive dimensional analysis, we have derived an upper bound on the coupling responsible for gaugino masses. This leads to a lower bound on the mass ratio $m_{3 / 2} / m_{1 / 2}$, which allows for a gravitino LSP in a large domain of parameter space. Some regions in parameter space are now allowed that were previously discarded in order to avoid a stau LSP. In particular, the compactification scale can coincide with the GUT scale even in the minimal scenario with vanishing Higgs soft 


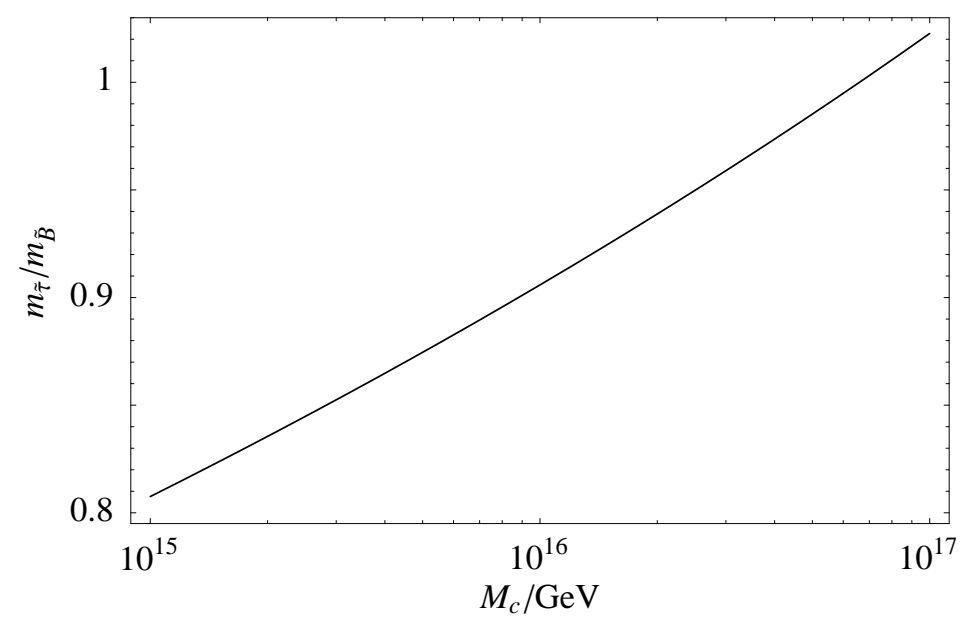

Figure 4: Ratio of stau and bino mass for $\tan \beta=10$ and $m_{1 / 2}\left(M_{c}\right)=400 \mathrm{GeV}$, calculated in a very rough approximation (see text).

masses. Fixing the gaugino mass, one can translate the result for the mass ratio into a lower bound on the gravitino mass. For a gluino mass of $1 \mathrm{TeV}$, we find $m_{3 / 2} \gtrsim 10 \mathrm{GeV}$.

A gravitino LSP can be naturally accompanied by a stau NLSP. Long-lived staus will then be observed at future colliders, and in their decays the gravitino may be discovered.

\section{Acknowledgments}

We would like to thank Adam Falkowski, Jörg Jäckel, Hyun Min Lee, Michael Ratz, Kai Schmidt-Hoberg, Michele Trapletti and Alexander Westphal for helpful discussions. This work has been supported by the "Impuls- und Vernetzungsfonds" of the Helmholtz Association, contract number VH-NG-006.

\section{References}

[1] H. Pagels, J. R. Primack, Phys. Rev. Lett. 48 (1982), 223.

[2] M. Viel, J. Lesgourgues, M. G. Haehnelt, S. Matarrese, A. Riotto, Phys. Rev. D71 (2005), 063534 [astro-ph/0501562].

[3] D. V. Nanopoulos, K. A. Olive, M. Srednicki, Phys. Lett. B127 (1983), 30.

[4] M. Y. Khlopov, A. D. Linde, Phys. Lett. B138 (1984), 265; J. R. Ellis, J. E. Kim, D. V. Nanopoulos, Phys. Lett. B145 (1984), 181.

[5] T. Moroi, H. Murayama, M. Yamaguchi, Phys. Lett. B303 (1993), 289.

[6] M. Bolz, W. Buchmüller, M. Plümacher, Phys. Lett. B443 (1998), 209 [hepph/9809381]; M. Fujii, T. Yanagida, Phys. Lett. B549 (2002), 273 [hep- 
ph/0208191]; M. Fujii, M. Ibe, T. Yanagida, Phys. Rev. D69 (2004), 015006 [hep$\mathrm{ph} / 0309064]$.

[7] W. Buchmüller, K. Hamaguchi, M. Ratz, Phys. Lett. B574 (2003), 156 [hep$\mathrm{ph} / 0307181]$.

[8] M. Fukugita, T. Yanagida, Phys. Lett. B174 (1986), 45.

[9] W. Buchmüller, P. Di Bari, M. Plümacher, Nucl. Phys. B643 (2002), 367 [hep$\mathrm{ph} / 0205349]$.

[10] I. V. Falomkin et al., Nuovo Cim. A79 (1984), 193 [Yad. Fiz. 39 (1984), 990].

[11] M. Kawasaki, K. Kohri, T. Moroi, Phys. Rev. D71 (2005), 083502 [astro$\mathrm{ph} / 0408426]$.

[12] M. Fujii, M. Ibe, T. Yanagida, Phys. Lett. B579 (2004), 6 [hep-ph/0310142]; J. R. Ellis, K. A. Olive, Y. Santoso, V. C. Spanos, Phys. Lett. B588 (2004), 7 [hepph/0312262]; J. L. Feng, S. Su, F. Takayama, Phys. Rev. D70 (2004), 075019 [hep-ph/0404231]; L. Roszkowski, R. Ruiz de Austri, JHEP 08 (2005), 080 [hep$\mathrm{ph} / 0408227$.

[13] J. L. Feng, A. Rajaraman, F. Takayama, Phys. Rev. Lett. 91 (2003), 011302 [hepph/0302215]; J. L. Feng, A. Rajaraman, F. Takayama, Phys. Rev. D68 (2003), 063504 [hep-ph/0306024].

[14] W. Buchmüller, K. Hamaguchi, M. Ratz, T. Yanagida, Phys. Lett. B588 (2004), 90 [hep-ph/0402179].

[15] K. Hamaguchi, Y. Kuno, T. Nakaya, M. M. Nojiri, Phys. Rev. D70 (2004), 115007 [hep-ph/0409248]; J. L. Feng, B. T. Smith, Phys. Rev. D71 (2005), 015004 [hepph/0409278]; K. Hamaguchi, A. Ibarra, JHEP 02 (2005), 028 [hep-ph/0412229]; A. Brandenburg, L. Covi, K. Hamaguchi, L. Roszkowski, F. D. Steffen, Phys. Lett. B617 (2005), 99 [hep-ph/0501287].

[16] M. Dine, A. E. Nelson, Y. Shirman, Phys. Rev. D51 (1995), 1362 [hep-ph/9408384]; M. Dine, A. E. Nelson, Y. Nir, Y. Shirman, Phys. Rev. D53 (1996), 2658 [hep$\mathrm{ph} / 9507378]$.

[17] H. P. Nilles, Phys. Rept. 110 (1984), 1.

[18] L. Randall, R. Sundrum, Nucl. Phys. B557 (1999), 79 [hep-th/9810155]; G. F. Giudice, M. A. Luty, H. Murayama, R. Rattazzi, JHEP 12 (1998), 027 [hep$\mathrm{ph} / 9810442]$.

[19] D. E. Kaplan, G. D. Kribs, M. Schmaltz, Phys. Rev. D62 (2000), 035010 [hepph/9911293]; Z. Chacko, M. A. Luty, A. E. Nelson, E. Ponton, JHEP 01 (2000), 003 [hep-ph/9911323]. 
[20] K. Inoue, M. Kawasaki, M. Yamaguchi, T. Yanagida, Phys. Rev. D45 (1992), 328.

[21] N. Arkani-Hamed, T. Gregoire, J. Wacker, JHEP 03 (2002), 055 [hep-th/0101233]; A. Hebecker, Nucl. Phys. B632 (2002), 101 [hep-ph/0112230].

[22] Z. Chacko, M. A. Luty, E. Ponton, JHEP 07 (2000), 036 [hep-ph/9909248].

[23] A. Hebecker, M. Trapletti, Nucl. Phys. B713 (2005), 173 [hep-th/0411131].

[24] K. Inoue, A. Kakuto, H. Komatsu, S. Takeshita, Prog. Theor. Phys. 68 (1982), 927; K. Inoue, A. Kakuto, H. Komatsu, S. Takeshita, Prog. Theor. Phys. 71 (1984), 413.

[25] G. Belanger, S. Kraml, A. Pukhov, Phys. Rev. D72 (2005), 015003 [hepph/0502079]; B. C. Allanach, S. Kraml, W. Porod, JHEP 03 (2003), 016 [hepph/0302102], http://cern.ch/kraml/comparison/.

[26] M. Schmaltz, W. Skiba, Phys. Rev. D62 (2000), 095005 [hep-ph/0001172];

M. Schmaltz, W. Skiba, Phys. Rev. D62 (2000), 095004 [hep-ph/0004210]. 